\title{
Análise de Exatidão entre MDEs: AW3D, SRTM-30m e Projeto SPMGG050
}

\author{
Accuracy Analysis between MDEs: AW3D, SRTM-30m and Project SPMGGO50
}

Análisis de Precisión entre MDEs: AW3D, SRTM-30m y Proyecto SPMGGO50

\author{
Antonio Santiago da Silva ${ }^{1}$ \\ Oberdan Rafael Pugoni Lopes Santiago ${ }^{2}$ \\ Claudionor Ribeiro da Silva ${ }^{3}$
}

\begin{abstract}
RESUMO: A partir do surgimento e disponibilização de base de Modelos Digitais de Elevação (MDE) de abrangência mundial e gratuitos, houve um expressivo aumento de trabalhos para a realização da modelagem de superfície da Terra. Contudo, a qualidade dos produtos gerados a partir desses dados e as escalas adequadas de uso ainda carecem de estudos mais detalhados. O presente trabalho visou analisar a precisão altimétrica de dados AW3D, SRTM e do projeto SPMGGO50 do IBGE na bacia hidrográfica do Ribeirão São Domingos (BHSD), município de Catalão/GO, avaliando a adequação desses dados às escalas 1:50.000, 1:100.000 e 1:250.000, observando a metodologia indicada pelo Padrão de Exatidão Cartográfica dos Produtos Cartográficos Digitais (PEC-PCD). Foram coletados 44 pontos de controle/validação em campo para a aferição dos modelos. Para tanto, foram calculados a tolerância vertical e o Erro médio quadrático dos modelos em relação aos pontos de validação. Os sensores SRTM e AW3D e o projeto SPMGGO50 atingiram a classe "B" na escala 1:100.000 e "A" na escala 1:250.000.
\end{abstract}

PALAVRAS-CHAVE: PEC-PCD. SRTM. AW3D. Projeto SPMGGO. Acurácia. MDE.

ABSTRACT: Since the emergence and availability of a worldwide and free base of Digital Elevation Models (DEM), there was a significant increase of works for the accomplishment of the Earth surface modeling. However, the quality of the products generated from these data and the appropriate scales of use still lack more detailed studies. This work aims at analyzing the altimetric precision of data

\footnotetext{
1 Mestrando em Geografia, Programa de Pós-Graduação em Geografia pela Universidade Federal de Uberlândia. Atualmente é técnico de laboratório e diagramador da Universidade Federal de Uberlândia. Av. João Naves de Ávila, 2160 - Campus Santa Mônica, Caixa Postal 38408-902, Uberlândia/MG. Email: asantiagogeo@gmail.com.

2 Mestrando em Geografia, Universidade Federal de Uberlândia, Programa de Pós-Graduação em Geografia. Av. João Naves de Ávila, 2160 - Campus Santa Mônica, Caixa Postal 38408-902, Uberlândia/MG. Gestor Ambiental, especialidade Engenharia Florestal, da Secretaria de Estado de Meio Ambiente e Desenvolvimento Sustentável - MG. Email: plsantiago@gmail.com.

${ }^{3}$ Doutorado em Ciências Geodésicas na Universidade Federal do Paraná. Pós-Doutorado na Universidade do Porto/Pt. - Professor Associado DE na Universidade Federal de Uberlândia (2011/Atual). Av. Amazonas, S/N, Campus Umuarama, Bloco 2E, Sala 119, CEP: 38400-902, Uberlândia/MG. E-mail: crs@ufu.br.
} 
AW3D, SRTM and the IBGE's SPGEGGO50 Project in the Ribeirão São Domingos Hydrographical Basin (BHSD), in Catalão, Goiás, evaluating the adequacy of these data at 1:50,000, 1:100,000 and 1:250,000 scales, observing the methodology suggested for the Cartographic Accuracy Standard for Digital Cartographic Products (PEC-PCD). 44 points of control/validation were collected in field to the assessing of the models. For this purpose, the vertical tolerance and the mean square Error of the models in relation to the validation points were calculated. The SRTM and AW3D sensors and Project SPMGGO50 reached category " $B$ " to the 1:100,000 and 1:250,000 scales.

KEYWORDS: PEC-PCD. SRTM. AW3D. Project SPMGGO. Accuracy. DME.

RESUMEN: A partir del surgimiento y disponibilización de los Modelos Digitales de Elevación (MDE) de alcance mundial y de carácter gratuito, hubo un aumento significativo en la realización de los trabajos para el modelaje de la superficie de la Tierra. De igual manera se observa que la calidad de los productos elaborados a partir de los datos y las escalas adecuadas de uso, todavía carecen de estudios más detallados. El presente trabajo se centra en analizar la precisión de los datos altimétricos de AW3D, SRTM y el Proyecto SPMGGO50 del IBGE en la cuenca de Ribeirão São Domingos, municipio de Catalão / GO, evaluando la posibilidad de realizar una adecuación de los datos a escalas 1: 50.000 , 1: 100.000 y 1: 250.000, observando la metodología indicada por el Padrón de Precisión Cartográfica de los Productos Cartográficos Digitales (PEC-PCD). Fueron recogidos los datos de 44 puntos de control / validación en campo para la medición de los modelos. Por ende, se hicieron los cálculos de tolerancia vertical y el Error medio cuadrático de los modelos en los puntos de validación. Los sensores SRTM y AW3D y el Proyecto SPMGGO50 alcanzaron la clasificación para considerarse dentro de la clase "B" en escala 1: 100.000 y "A" 1: 250.000.

PALABRAS-CLAVE: PEC-PCD. SRTM. AW3D. Proyecto SPMGGO. Precisión. MDE.

\section{INTRODUÇÃO}

O avanço tecnológico tem propiciado o desenvolvimento de sensores que facilitam a obtenção de dados espaciais, bem como a popularização de computadores com maiores capacidades de armazenamento e de processamento de dados. Dessa forma, com o grande volume de dados gerados e com o aumento da demanda por dados mais precisos, surge a necessidade de validação dessas novas fontes, especialmente dos dados altimétricos, com base nas normas em vigência no país.

Os estudos sobre a geração de Modelos Digitais de Elevação (MDE), a partir de sensores orbitais, estão amplamente desenvolvidos e consolidados; contudo, a análise da qualidade desse produto, em diferentes escalas, ainda é incipiente. Uma das principais fontes desses dados ainda é a base gerada pela missão SRTM (Shuttle Radar Topography Mission). A SRTM foi uma missão realizada em fevereiro de 2000 , liderada pela NASA (National Aeronauticsand Space Administration) e NIMA (National Imagery and Mapping Agency), para a geração de modelos altimétricos na área continental, com resolução de um e três segundos de arco, ou seus equivalentes métricos aproximados de 30 e 90 metros, e 10 metros de exatidão na medida vertical, 
com o nível de confiança de $90 \%$. O dado pode ser obtido, sem custos, no site: http://earthexplorer.usgs.gov.

O projeto ALOS World 3D - 30m (AW3D), planejado com o satélite japonês Advanced Land Observing Satellite-2, (ALOS-2, "DAICHI-2"), surgiu como alternativa de geração de dados altimétricos, no âmbito global. O ALOS-2 possui a bordo o sensor Stereo Mapping (PRISM), que gera dados com resolução espacial de 0,15 segundos de arco na horizontal (latitude e longitude), ou seja, aproximadamente cinco metros, e ainda imagem ortoretificada (ORI). As imagens AW3D-PRISM, com um segundo de arco (cerca de 30 metros) de resolução na horizontal e exatidão altimétrica de cinco metros, obtidas entre os anos de 2015-2016, são disponibilizadas gratuitamente pela agência japonesa JAXA (2016).

O Instituto Brasileiro de Geografia e Estatística também fornece MDE de algumas regiões do Brasil, gerado a partir de fotografias aéreas. É uma importante fonte de informações espaciais altimétricas de qualidade. Nesse sentido, enquadra-se o Modelo Digital de Elevação MI-2415-1; MI-2415-2, gerado na escala de 1:50.000, do Projeto SPMGGO50 (São Paulo - Minas Gerais - Goiás), cuja fonte, de acordo com os metadados disponibilizados pelo IBGE, são obtidos de fotografias aéreas com escala aproximada de 1:35.000, com resolução espacial de $0.7 \mathrm{~m}$ e com acurácia altimétrica média de cinco metros (IBGE, 2012).

Conforme Santillan e Makinano-Santillan (2016), o dado AW3D superou o dado SRTM de 30 metros, em termos de precisão vertical, devido aos menores erros médios e valores do RMSE. Contudo, a utilização dos modelos altimétricos derivados desses dados (SRTM e PRISM) deve ser cuidadosa quanto à precisão necessária e à escala cartográfica usada, pois há limitação das informações e erros aleatórios bem superiores aos valores médios em locais aleatórios (CHAGAS et al. 2010; SOUZA, 2015). Portanto, torna-se necessário examinar se a precisão/exatidão são compatíveis com a escala e precisão desejadas.

Preocupado com a acurácia de dados digitais, Valeriano (2002) descreveu métodos de avaliação da qualidade desses dados, testando modelos gerados a partir de dados SRTM com dados digitalizados de cartas 1:50.000, avaliando a qualidade visual e descritiva dos modelos e aplicando técnicas geoestatísticas.

Miceli et al. (2010) analisaram a acuidade posicional e geométrica de ambos os modelos (SRTM e PRISM), destacando padrões e cuidados para aumentar o grau de confiabilidade no processo de análise e produção de dados espaciais. Esses autores fizeram, mais precisamente, uma avaliação comparativa dos dados derivados dos modelos de elevação ASTER/PRISM e do SRTM, em relação às suas precisões horizontal e vertical.

No presente trabalho, o objetivo é comparar e avaliar a acurácia altimétrica dos dados SRTM, AW3D (PRISM) e projeto SPMGGO50 nas escalas de 1:50.000, 1:100.000 e 1:250.000, considerando o padrão determinado pelo Ministério da Defesa (BRASIL, 2015). 


\section{MATERIAL E MÉTODO}

\section{Área de estudo}

O estudo foi desenvolvido na bacia hidrográfica do Ribeirão São Domingos (BHSD), afluente do rio São Marcos, município de Catalão, Goiás. O centro da BHSD está localizado nas seguintes coordenadas geográficas: $47^{\circ} 46^{\prime} 848^{\prime \prime} \mathrm{S}, 1^{\circ} 04^{\prime} 015^{\prime \prime} \mathrm{O}$. A BHSD apresenta uma área de 83 quilômetros quadrados e é constituída de relevo mais plano com elevações acima de 800 metros e áreas mais declivosas, à jusante desse ribeirão, com aproximadamente 700 metros de altitude (Figura 1).

Figura 1 - Localização da bacia hidrográfica Ribeirão São Domingos; distribuição de pontos coletados a campo; e comparação entre os MDE's Projeto SPMGGO, SRTM-30 e AW3D

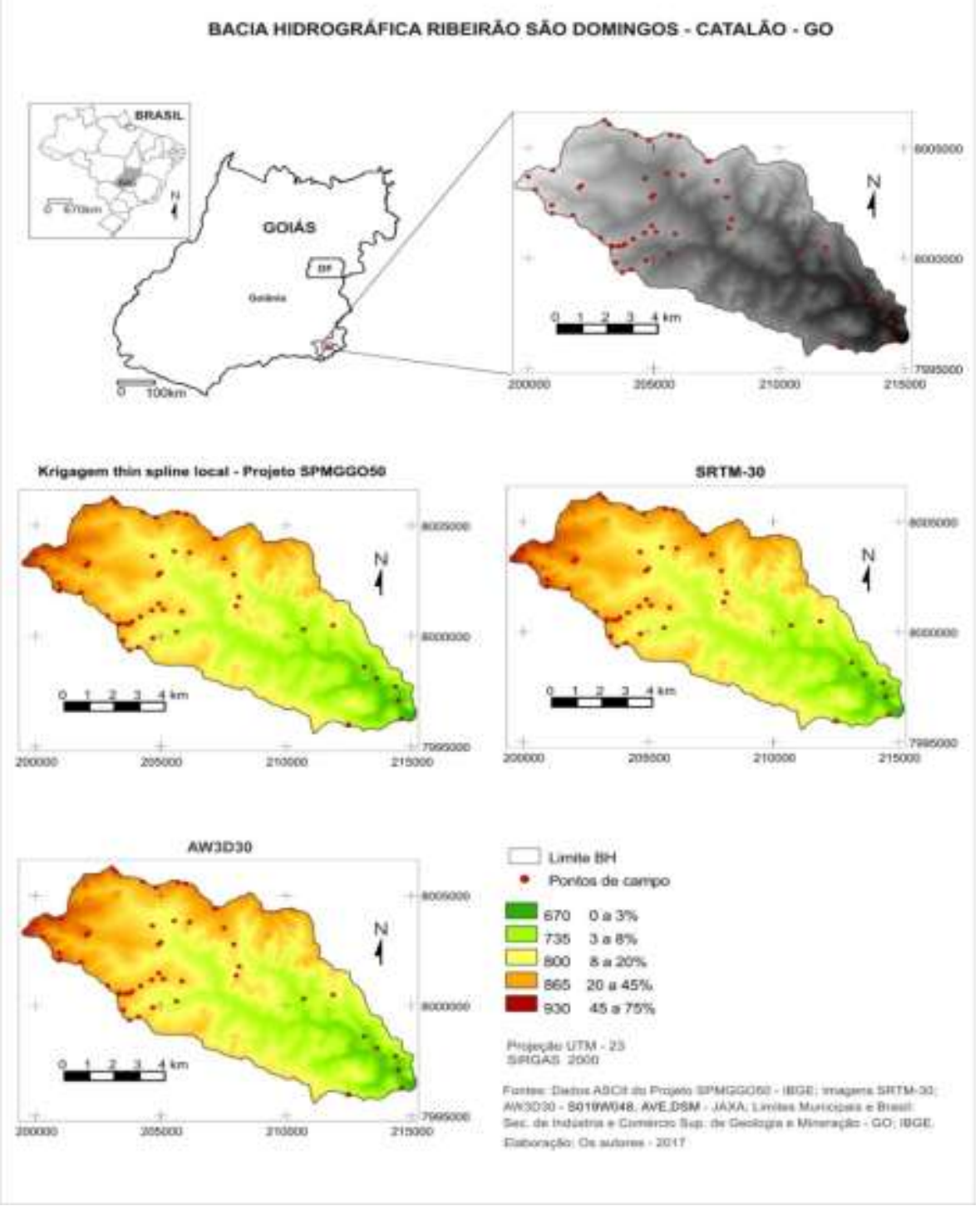

Fonte: Os autores (2017). 


\section{Material usado e preparação da base de dados}

Para a realização dos experimentos, foram utilizadas uma cena do AW3D e outra do SRTM, ambas com 30 metros de resolução espacial (grid horizontal), e com cinco e 10 metros de acurácia vertical, respectivamente, bem como um grid de pontos XYZ'.ASCII', referentes a um Modelo Digital de Elevação, denominados de MI-2415-1 e MI-2415-2, na escala de 1:50.000, pertencentes ao projeto SPMGGO50 do IBGE(2012).

Ambas as cenas foram reprojetadas para a projeção Universal Transversa de Mercator (UTM), Zona $23 S$ e referencial geodésico Sirgas 2000 e o grid '.ASCIl' já foi modelado nessa mesma projeção. Os MDE's foram gerados no software livre SAGA com interpolação (Kriging and thin plate spline), através de grid de pontos XYZ '.ASCIl'. O MDE do projeto SPMGGO50 foi gerado com o tamanho de células equivalente às equidistâncias de 20, $50 \mathrm{e}$ 100 metros, para escalas 1:50.000, 1:100.000, 1:250.000, respectivamente. A escolha da interpolação por krigagem foi feita com base na pesquisa de Valeriano (2002), que afirma que os MDE's gerados por esse método produzem formas de relevo e feições de drenagem mais realistas do que os interpoladores lineares e o Inverso do Quadrado da Distância (IQD).

$O$ arquivo vetorial do limite da BHSD foi obtido a partir de MDE gerado com os dados do projeto SPMGGO, por ser de melhor qualidade geométrica. Nesse processo, foi utilizado o menu de processamento do QGIS 2.8.4 (QGIS, 2015), no módulo GRASS (r.watershed). Finalmente, os recortes da área de interesse para o estudo foram corrigidos, geometricamente, com base em cartas topográficas da DSG, na escala 1:100.000.

Para a análise da acurácia, adotou-se o Padrão de Exatidão Cartográfica dos Produtos Cartográficos Digitais (PEC-PCD), de acordo com a Especificação Técnica dos Produtos de Conjuntos de Dados Geoespaciais (ET-PCDG) (BRASIL, 2015). Segundo Martin (2016), essa norma foi desenvolvida baseada no Decreto-lei n 89.817, de 20 de junho de 1984, sendo que, para as escalas não contempladas nesse dispositivo legal, foram feitas extrapolações sem alterar os valores em milímetros previstos do PEC Planimétrico e do PEC Altimétrico. Na Tabela 1 estão dispostos os valores do PEC Altimétrico dos pontos cotados e dos Modelos Digitais do Terreno (MDT), de Elevação (MDE) e de Superfície (MDS), para a produção de Produtos Cartográficos Digitais.

Para elaborar a base de referência, foram coletados em campo 44 pontos $(x, y, z)$ distribuídos na área do estudo. Para aleatorizar a amostra, procedeu-se a um sorteio da sequência dos pontos, dispostos conforme Figura 1. A altimetria desses pontos foi coletada com um altímetro eletrônico, marca Suunto Escape E203, com precisão de 1-3 metros e a planimetria medida com um GPS Garmin Etrex Legend $\mathrm{H}$, cuja precisão média alcançada em campo foi de três metros, conforme aferição realizada para ambos os equipamentos. 
Foram aferidas a altimetria e a planimetria, com duas horas de observações nas Estações Geodésicas RN 3230-J e 8055922, localizadas no município de Catalão. As observações foram realizadas em dois dias.

Tabela 1 - Padrão de Exatidão Cartográfica Altimétrica dos Pontos Cotados e do MDT, MDE e MDS para a produção de Produtos Cartográficos Digitais, segundo a PEC-PCD / ETPCDG

\begin{tabular}{|c|c|c|c|c|c|c|c|c|}
\hline \multirow{2}{*}{$\begin{array}{c}\text { PEC - PCD } \\
\text { (3) }\end{array}$} & \multicolumn{2}{|c|}{ 1:1.000 } & \multicolumn{2}{|c|}{$1: 2.000$} & \multicolumn{2}{|c|}{$1: 5.000$} & \multicolumn{2}{|c|}{$1: 10.000$} \\
\hline & $\begin{array}{l}\text { PEC } \\
(\mathrm{m})\end{array}$ & $\begin{array}{l}\mathrm{EP} \\
(\mathrm{m})\end{array}$ & $\begin{array}{l}\text { PEC } \\
(\mathrm{m})\end{array}$ & $\begin{array}{l}E P \\
(m)\end{array}$ & $\begin{array}{l}\text { PEC } \\
(\mathrm{m})\end{array}$ & $\begin{array}{l}E P \\
(m)\end{array}$ & $\begin{array}{c}\text { PEC } \\
(\mathrm{m})\end{array}$ & $\begin{array}{l}\mathrm{EP} \\
(\mathrm{m})\end{array}$ \\
\hline$A$ & 0,27 & 0,17 & 0,27 & 0,17 & 0,54 & 0,34 & 1,35 & 0,84 \\
\hline$B$ & 0,50 & 0,33 & 0,50 & 0,33 & 1,00 & 0,66 & 2,50 & 1,67 \\
\hline C & 0,60 & 0,40 & 0,60 & 0,40 & 1,20 & 0,80 & 3,00 & 2,00 \\
\hline $\mathrm{D}$ & 0,75 & 0,50 & 0,75 & 0,50 & 1,50 & 1,00 & 3,75 & 2,50 \\
\hline $\begin{array}{l}\text { PEC - PCD } \\
\text { (3) }\end{array}$ & \multicolumn{2}{|c|}{$1: 25.000$} & \multicolumn{2}{|c|}{$1: 50.000$} & \multicolumn{2}{|c|}{$1: 100.000$} & \multicolumn{2}{|c|}{$1: 250.000$} \\
\hline$A$ & 2,70 & 1,67 & 5,50 & 3,33 & 13,70 & 8,33 & 27,00 & 16,67 \\
\hline$B$ & 5,00 & 3,33 & 10,00 & 6,66 & 25,00 & 16,66 & 50,00 & 33,33 \\
\hline C & 6,00 & 4,00 & 12,00 & 8,00 & 30,00 & 20,00 & 60,00 & 40,00 \\
\hline $\mathrm{D}$ & 7,50 & 5,00 & 15,00 & 10,00 & 37,50 & 25,00 & 75,00 & 50,00 \\
\hline
\end{tabular}

Fonte: Adaptado pelos de: (BRASIL, 2015).

Para validação dos MDEs foi realizada uma análise estatística por meio de uma comparação entre os pontos observados na base de referência e seus correspondentes obtidos nos MDEs. Para isso, foram geradas três tabelas de pontos $(x, y, z)$ correspondentes aos modelos AW3D, SRTM30m e projeto SPMGGO50, e uma quarta tabela com os dados coletados em campo com o altímetro eletrônico (base de referência). A coleta dos pontos nos MDEs, a partir das coordenadas dos pontos de referência, foi realizada com base no método do vizinho mais próximo, ou seja, a altimetria do ponto mais próximo da coordenada de referência foi coletada para preenchimento da tabela do respectivo MDE. Essa tarefa foi executada no software QGIS 2.8.4. (QGIS, 2015).

Em seguida, aplicou-se o teste Qui-quadrado para verificação de independência dos dados, utilizando o software Microsoft Excel, e os testes Shapiro-Wilk e KolmogorovSmirnov, para verificação da normalidade, a partir do complemento 'ActionStat' do Excel. 
Comprovadas a independência e a normalidade dos dados, foram calculados os erros médios quadrados dos desvios (RMS), conforme Equação 1, para os valores de altitude observados de cada sensor, em relação aos valores coletados em campo com altímetro eletrônico,

$$
R M S=\sqrt[2]{\frac{\sum_{i=1}^{n}\left(\widehat{y}_{l}-y_{i}\right)^{2}}{n}}(1)
$$

onde $\widehat{y}_{l}$ é o valor observado (valor da base de referência) na i-ésima observação e $y_{i}$ é o valor predito (valor correspondente à i-ésima observação, obtido no MDE).

Foi verificado se o valor do RMS das discrepâncias médias é menor ou igual ao valor do EP, para a escala e classe em teste e para a classificação dos MDEs, seguindo as premissas estabelecidas pelo PEC-PCD. Por fim, foi realizado o teste $z$ de hipóteses para testar diferenças entre as médias dos erros de cada produto.

\section{RESULTADOS E DISCUSSÃO}

Como não há informações objetivas sobre o Datum vertical do projeto SPMGG050 nos metadados, foi realizada consulta à Coordenação de Cartografia do IBGE, a qual informou que o produto está referenciado ao Datum de Imbituba, assim como o altímetro utilizado. Os dados dos modelos AW3D30 e SRTM foram disponibilizados no modelo de geoide EGM96 (JAXA, 2016; UNITED STATES GEOLOGICAL SURVEY, 2016). Conforme Luz, Freitas e Dalazoana (2002), a discrepância entre o modelo EGM96 e o Datum Vertical Brasileiro é cerca de 10 centímetros. Logo, para os fins deste estudo, tal erro não é considerável para as escalas utilizadas. Na Tabela 2 estão relacionados os pontos coletados em campo e que foram utilizados como referência nas análises.

Segundo o Ministério da Defesa, para um produto cartográfico digital ser aceito como produto de Referência do Sistema Cartográfico Nacional, deve atender a dois critérios simultaneamente: a) $90 \%$ dos pontos coletados no produto em questão devem ter valores de erro abaixo ou iguais ao PEC-PCD; b) os valores de Erro Padrão (EP) também devem ser iguais ou inferiores aos de referência (BRASIL, 2015).

O erro médio quadrático (RMS) encontrado para o sensor AW3D foi de 12,45 metros enquanto que para o sensor SRTM foi de 11,69 metros. Com esses valores não foram alcançadas as condições para classificação na escala 1:50.000. Dessa forma, ambos os sensores possuem o valor de RMS menor que o EP da PEC-PCD para a classe "B", na escala 1:100.000, cujo valor é de 16,66 metros. Ambos os sensores, na mesma escala, atenderam à condição de $90 \%$ do erro dos pontos serem menores ou 
iguais à tolerância de 25 metros, atingindo $100 \%$ em ambos. Portanto, foi possível classificá-los na classe "B", para a escala 1:100.000. Para a escala 1:250.000, o sensor AW3D atendeu à condição de RMS menor ou igual a 16,67 metros e apresentou 100\% dos erros menor ou iguais a 27 metros. Dessa forma, pode ser classificado na classe PEC-PCD "A" para a escala de 1:250.000. Da mesma maneira, o sensor SRTM apresentou RMS menor ou igual a 16,67 metros e $100 \%$ dos erros menores ou iguais a 27 metros. Logo, também, atende aos critérios da classe PEC-PCD "A" para a escala de 1:250.000. Portanto, foi possível classificá-los na classe "A", para a escala 1:100.000, como resumido na Tabela 3.

Tabela 2 - Pontos coletados em campo

\begin{tabular}{cccc}
\hline Pontos & $\mathbf{E}(\mathbf{m})$ & $\mathbf{N}(\mathbf{m})$ & $\begin{array}{c}\mathbf{Z} \\
\text { ortométrico } \\
(\mathbf{m})\end{array}$ \\
\hline 1 & 199994,412 & 8003707,854 & 910 \\
\hline 2 & 200983,018 & 8003990,148 & 923 \\
\hline 3 & 202127,35 & 8003297,792 & 895 \\
\hline 4 & 204674,207 & 8003622,04 & 832 \\
\hline 5 & 204904,755 & 8002765,772 & 843 \\
\hline 6 & 205541,278 & 8003838,458 & 805 \\
\hline 7 & 206150,576 & 8003781,999 & 850 \\
\hline 8 & 206010,773 & 8005537,91 & 888 \\
\hline 9 & 204299,361 & 8005612,202 & 885 \\
\hline 10 & 203220,013 & 8006077,655 & 902 \\
\hline 11 & 200275,718 & 8003102,353 & 908 \\
\hline 12 & 200941,809 & 8002402,107 & 895 \\
\hline 13 & 200957,303 & 8002041,638 & 918 \\
\hline 14 & 201788,061 & 8001960,861 & 900 \\
\hline 15 & 202882,478 & 8000904,673 & 866 \\
\hline 16 & 203580,466 & 8000542,324 & 884 \\
\hline 17 & 203756,126 & 7999354,635 & 850 \\
\hline 18 & 203512,691 & 7999818,683 & 851 \\
\hline 19 & 203313,52 & 8000510,067 & 878 \\
\hline 20 & 203357,11 & 8000575,953 & 874 \\
\hline 21 & 203757,426 & 8000546,948 & 869 \\
\hline 22 & 204194,054 & 8000878,678 & 843 \\
\hline
\end{tabular}

Fonte: Os autores (2017).

\begin{tabular}{cccc}
\hline Pontos & $\mathbf{E}(\mathbf{m})$ & $\mathbf{N}(\mathbf{m})$ & $\begin{array}{c}\mathbf{Z} \\
\text { ortométrico } \\
(\mathbf{m})\end{array}$ \\
\hline 23 & 204633,717 & 8001161,588 & 846 \\
\hline 24 & 205107,622 & 8001207,003 & 855 \\
\hline 25 & 204929,254 & 8001466,079 & 847 \\
\hline 26 & 205856,258 & 8001109,03 & 846 \\
\hline 27 & 205634,107 & 8000203,013 & 785 \\
\hline 28 & 204114,369 & 7999491,971 & 839 \\
\hline 29 & 203047,995 & 8006263,11 & 892 \\
\hline 30 & 203216,325 & 8006069,531 & 889 \\
\hline 31 & 204298,051 & 80056008,394 & 872 \\
\hline 32 & 204817,174 & 8005340,629 & 873 \\
\hline 33 & 205684,183 & 8005584,806 & 878 \\
\hline 34 & 207151,318 & 8004402,229 & 879 \\
\hline 35 & 207232,876 & 8004414,013 & 881 \\
\hline 36 & 207531,631 & 8003516,007 & 865 \\
\hline 37 & 208127,894 & 8001774,433 & 827 \\
\hline 38 & 208016,786 & 8001363,264 & 826 \\
\hline 39 & 210706,967 & 8000311,218 & 742 \\
\hline 40 & 211881,881 & 8000490,118 & 808 \\
\hline 41 & 213622,88 & 7998089,974 & 711 \\
\hline 42 & 214483,712 & 7997074,78 & 702 \\
\hline 43 & 214617,978 & 7996262,45 & 720 \\
\hline 44 & 212497,009 & 7995996,157 & 765 \\
\hline & & & \\
\hline
\end{tabular}

Da mesma maneira, o MDE projeto SPMGGO50 apresentou RMS menor que 16,66 metros (RMS calculado foi de 15,07 metros) e $100 \%$ dos erros menores ou iguais a 25 metros. Logo, também atende aos critérios da classe PEC-PCD "C" para a escala 1 : 
100.000. Já para a escala 1:250.000 atingiu a classe "A", cujos valores de referência para são 16,67 e 27 metros, respectivamente. Os dados apresentados pelo modelo não permitiu sua classificação na escala 1:50.000.

Tabela 3 - Classificação da acurácia posicional dos MDE's AW3D (1), SRTM (2), projeto SPMGGO50 (3), utilizando como padrão o Decreto n 89.817/ET-ADGV para a escala 1:50.000, 1:100.000 e 1:250.000

\begin{tabular}{c|c|c|c}
\hline Classe PEC-PCD & $1: 50.000$ & $1: 100.000$ & $1: 250.000$ \\
\hline A & & & (1), (2) e (3) \\
\hline B & & $(1),(2) \mathrm{e} \mathrm{(3)}$ & \\
\hline C & & & \\
\hline
\end{tabular}

Fonte: Os autores (2017).

A partir da análise da dispersão dos erros dos pontos dos sensores AW3D, SRTM e projeto SPMGGO50 (Figura 2), é possível verificar que há um comportamento semelhante entre AW3D e SRTM.

O projeto SPMGGO50 possui a fonte de dados com maior precisão, porém apresentou o pior desempenho ao alcançar RMS igual a 15,07, atingindo apenas a classe "B" na escala de 1:100.000. Supõe-se que ocorreram algumas anomalias no projeto. De acordo com o IBGE (2012), áreas de sombra, desníveis ou descontinuidades, tabuleiros de forma triangular e padrões de valores que não correspondem ao terreno podem afetar os resultados. Porém, essas mesmas condições podem afetar os outros produtos também. Dessa forma, sugere-se a análise em outras áreas e condições geomorfológicas diversas para averiguar o desempenho dos dados oficiais.

Por fim, foi realizado o teste de hipóteses descrito na Figura 3. Os produtos oriundos dos modelos AW3D e SRTM não apresentaram diferença estatística entre si. No entanto, ambos foram classificados como diferentes do modelo do IBGE, apresentando características superiores, conforme a classe atingida para o PEC-PCD. Embora o sensor AW3D tenha exatidão altimétrica nominal de 5 metros, apresentou a mesma qualidade cartográfica que o sensor SRTM $30 \mathrm{~m}$, que possui exatidão altimétrica nominal de aproximadamente 10 metros. 
Figura 2 - Dispersão dos erros dos pontos nos MDEs: a) AW3D, b) SRTM e c) SPMGGO Dispersão dos erros (m) - ALOS2 3D

a)

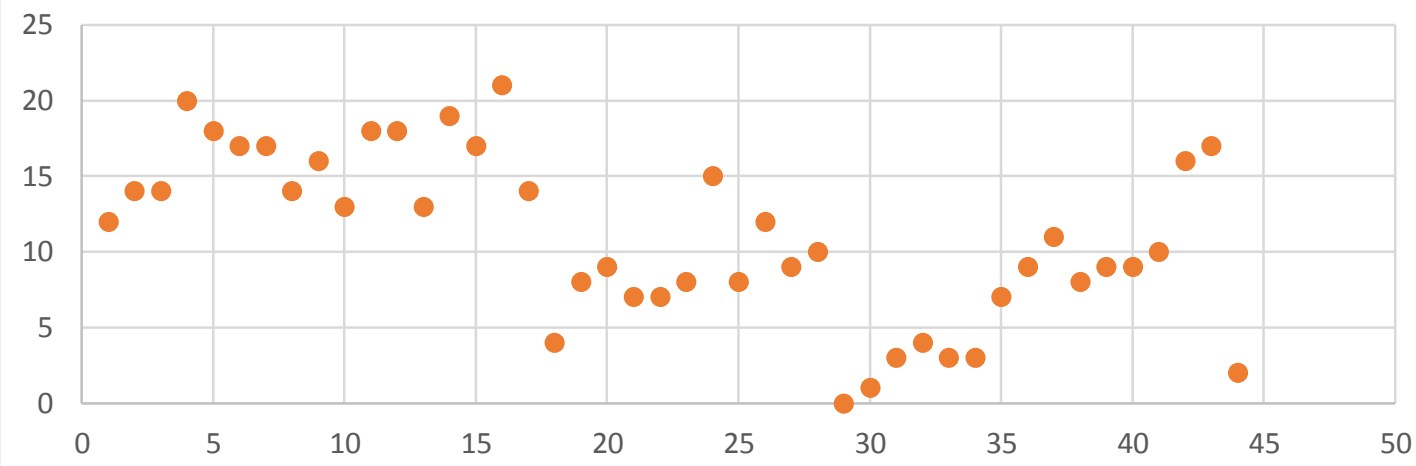

Dispersão dos erros (m) - SRTM

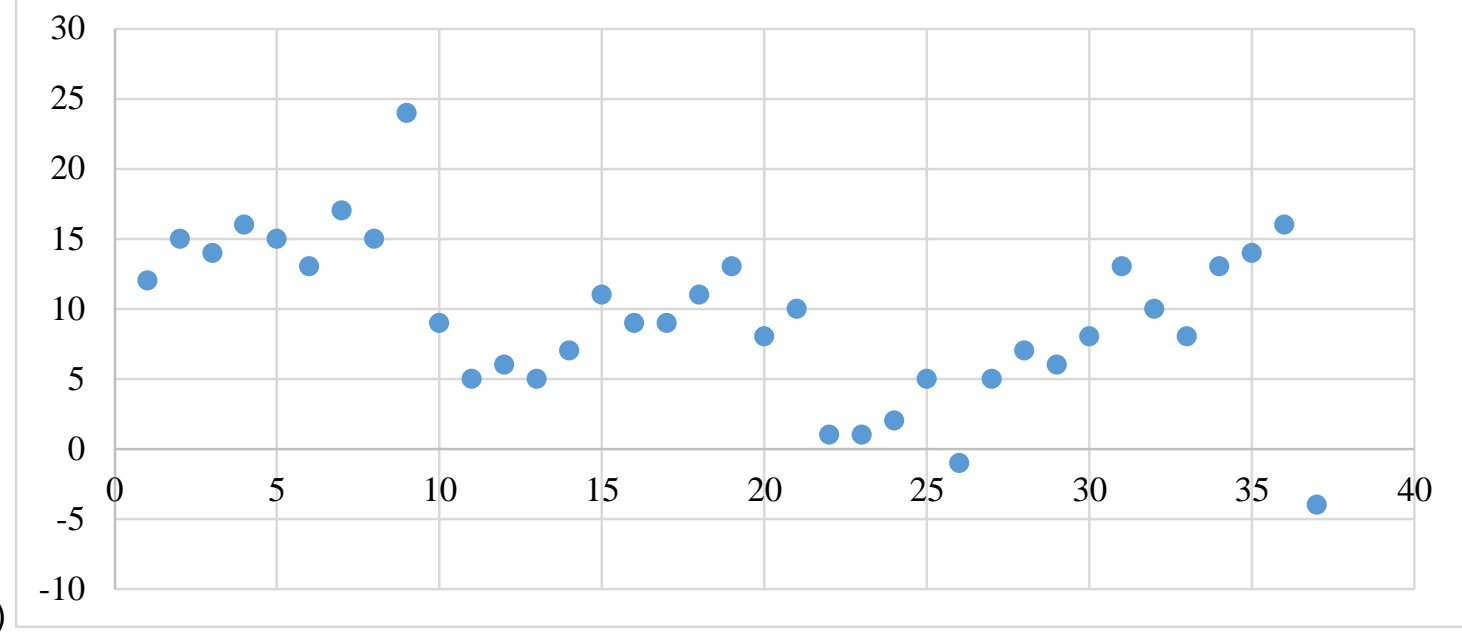

b)

Dispersão dos erros (m) - SPMGG050

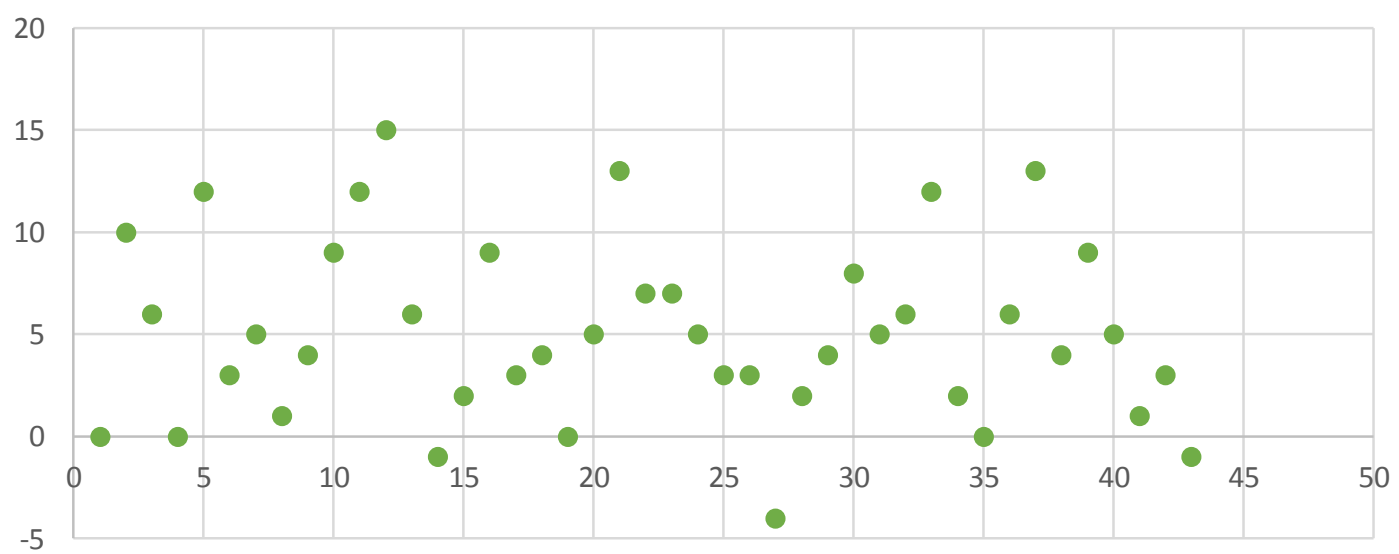

c)

Fonte: Os autores (2017). 
Figura 3 - Testes de hipóteses de médias

\begin{tabular}{|c|c|c|c|}
\hline \multicolumn{5}{|c|}{ Teste de hipótese "z" ( $\mathbf{a}=\mathbf{0 , 0 5})$} \\
\hline H0 & AW3D $\times$ SRTM & AW3D x IBGE & SRTM x IBGE \\
\hline H1 & AW3D = SRTM & AW3D = IBGE & SRTM = IBGE \\
\hline |Z calculado| & AW3D $\neq$ SRTM & AW3D $\neq$ IBGE & SRTM ₹ IBGE \\
\hline Z crítico & 0,731 & 2,010 & 2,692 \\
\hline Hipótese aceita & 1,960 & 1,960 & 1,960 \\
\hline
\end{tabular}

Fonte: Os autores (2017).

\section{CONSIDERAÇÕES FINAIS}

Este artigo introduziu visões gerais e análise comparativa entre os MDEs elaborados a partir dos dados AW3D, SRTM e SPMGGO50, com base no PEC-PCD. Imagens AW3D ainda são pouco utilizadas no meio acadêmico e técnico, em comparação ao SRTM, que possui técnicas e métodos de processamento mais consolidados. O presente estudo aponta que ambos os sensores do AW3D e SRTM podem ser considerados uma boa fonte de dados para trabalhos com escalas médias e pequenas (menores ou iguais a 1:100.000).

Sugerem-se estudos para a avaliação entre os dois sensores para terrenos mais declivosos, onde se acredita que a exatidão altimétrica do sensor AW3D pode ter desempenho melhor que o SRTM, dado que sua acurácia altimétrica nominal é de 5 metros. O projeto de iniciativas entre a JAXA e o setor privado, utilizando conjunto de dados MDE de resolução espacial de 30 metros e altimetria de cinco metros sobre área global, é capaz de contribuir com diversas aplicações, desde que observada a sua precisão.

O projeto SPMGGO50 é uma das fontes de informações altimétricas mais precisas disponibilizadas gratuitamente, e pode ser mais explorado nos estudos acadêmicos. No entanto, sugerem-se outros estudos comparativos para condições geomorfológicas diferentes, principalmente em terrenos declivosos para teste de exatidão.

\section{REFERÊNCIAS}

BRASIL. Ministério da Defesa. Exército Brasileiro. Diretoria do Serviço Geográfico. Especificação técnica para a aquisição de dados geoespaciais vetoriais: ET-ADGV defesa FT 2015. Disponível em:

<http://www.geoportal.eb.mil.br/index.php/inde2?id=140>. Acesso em: 9 nov. 2016.

CHAGAS, C. S. et. al. Avaliação de modelos digitais de elevação para aplicação em um mapeamento digital de solos. Revista Brasileira de Engenharia Agrícola e Ambiental, Campina Grande, PB, v. 14, n. 2, p. 218-226, 2010. Disponível em:

<http://www.scielo.br/pdf/rbeaa/v14n2/v14n02a14.pdf>. Acesso em: 25 jan. 2017. 
IBGE. Projeto SPMGGO 1:50.000 (SPMGG050). 2012. Disponível em:

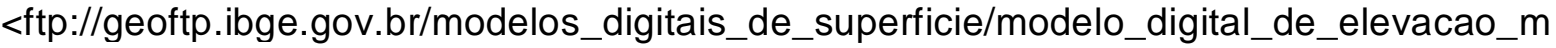
de/spmggo50>. Acesso em: 30 nov. 2016.

JAXA. ALOS Global Digital SurfaceModel "ALOS World 3D - 30m" (AW3D). Disponível em: <http://www.eorc.jaxa.jp/ALOS/en/AW3D/index.htm>. Acesso em: 9 nov. 2016.

LUZ, R. R.; FREITAS, S. R. C.; DALAZOANA, R. Acompanhamento do Datum Altimétrico Imbituba através das redes altimétrica e maregráfica do Sistema Geodésico Brasileiro.

Anais... In: CONGRESSO INTERNACIONAL DE CIENCIAS DE LA TIERRA, 7., 2002,

Santiago, CL. Santiago: IGM, 2002. Disponível em:

$<$ https://ww2.ibge.gov.br/home/geociencias/geodesia/artigos/2002-

Acompanhamento_Imbituba_atraves_Redes_SGB.pdf>. Acesso em: 20 jun. 2018.

MARTIN, L. PEC, o que é e como aplicar?: [parte1]. 2016. Disponível em:

<http://www.engesat.com.br/pec-o-que-e-e-como-aplicar-1/>. Acesso em: 30 jan. 2017.

MICELI, B. S. et. al. Comparação da avaliação vertical de modelos digitais de elevação para médias e pequenas escalas em diferentes configurações topográficas. In:

SIMPÓSIO BRASILEIRO DE CIÊNCIAS GEODÉSICAS E TECNOLOGIAS DA

GEOINFORMAÇÃO, 3., 210, Recife, PE. Anais... Recife: UFPE, 2010, p. 1 - 7.

Disponível em:

<https://www.ufpe.br/cgtg/SIMGEOIII/IIISIMGEO_CD/artigos/CartografiaeSIG/SIG/A_191 .pdf>. Acesso em: 30 jan. 2017.

QGIS. Development Team 2015. QGIS: versão 2.8.4 Wien. Disponível em: <http://www.qgis.org/pt_BR/site/index.html>. Acesso em: 2 nov. 2016.

SANTILLAN, J.; MAKINANO-SANTILLAN, M. Vertical accuracy assessment of 30-M resolution alos, aster, and SRTM global dems over northeastern Mindanao, Philippines. In: ISPRS CONGRESS: THE INTERNATIONAL ARCHIVES OF THE PHOTOGRAMMETRY, REMOTE SENSING AND SPATIAL INFORMATION SCIENCES, 23., 2016, Prague, Czech Republic. Analls... Prague: ISPRS, 2016. v. XLI-B4, p. 149-155. Disponível em: $<$ http://www.int-arch-photogramm-remote-sens-spatial-inf-sci.net/XLI-B4/149/2016/isprsarchives-XLI-B4-149-2016.pdf >. Acesso em: 30 jan. 2017.

SOUZA. J. O. P. Análise da precisão altimétrica dos modelos digitais de elevação para área semiárida do nordeste brasileiro. Revista do Departamento de Geografia, São Paulo, v. 30, p. 56-64, 2015. Disponível em: <http://www.revistas.usp.br/rdg/article/view/98555>. Acesso em: 30 jan. 2017.

UNITED STATES GEOLOGICAL SURVEY. Shuttle Radar Topography Mission (SRTM)1 Arc-Second Global. Disponível em: <https://ta.cr.usgs.gov/SRTM1Arc>. Acesso em: 2 nov. 2016.

VALERIANO, M. M. Modelos digitais de elevação de microbacias elaborados com krigagem. São José dos Campos: INPE, 2002. Inpe-RPQ/736.

Recebido: outubro de 2017. Aceito: julho de 2018. 